\title{
A Novel Dual-Band Frequency Selective Surface Absorber
}

\author{
Mudassar Abdin, Umair Rafique*, Fahad Malik, Shahzaib Qasim, M. Arif Khan, M. Mansoor Ahmed
}

Department of Electronic Engineering, Mohammad Ali Jinnah University, Islamabad, 44000, Pakistan

\begin{abstract}
In this paper, a dual-band Frequency Selective Surface (FSS) absorber is presented for Wireless Local Area Network (W LAN) security. The FSS absorber is designed for two frequencies of interest, i.e., $2.45 \mathrm{GHz}$ (lower band) and $5.25 \mathrm{GHz}$ (upper band), respectively. FSS design consists of two layers; first layer consists of conducting square-loop elements while second layer consists of resistive square-loop elements, placed in-front of a conducting FSS layer. The proposed FSS design provides good frequency stability for oblique incidences for perpendicular (TE) and parallel (TM) polarizations. It provides better absorption rate for $2.45 \mathrm{GHz}$ and $5.25 \mathrm{GHz}$ frequency bands and allow the transmission of other microwave frequencies. Also, the proposed FSS design reduces multipath fading of additional WLAN signals which occurred due to the placement of conducting FSS layer. Presented results demonstrate that the proposed FSS design has a stable frequency response for the desired frequency bands for TE and TM polarizations with good absorption rate when the incident wave angle varied from $0^{0}$ to $45^{\circ}$.
\end{abstract}

Keywords Dual-Band, Frequency Selective Surface (FSS), Wireless Local Area Network (W LAN), Oblique Incidences, Perpendicular Polarization, Parallel Polarization

\section{Introduction}

Now-a-days, the use of Frequency Selective Surfaces (FSSs) is becoming popular as a free-space filter design. It was first demonstrated by Ben A. Munk and used the presented technology for airborne applications[1]. Many researchers proposed FSS designs for other microwave related problems, in which radome design for radar applications gets popularity[2]. Other applications associate d with FSSs are radar and analog absorbers[3-4], High Impedance Surfaces (HISs) for the enhancement of bandwidth and gain of an antenna[5], band-pass filter design for the transmission of radio frequencies through energy saving windows [6], resonator design or Electro magnetic Band-Gap (EBG) structure for the coupling reduction between microstrip patch antennas[7], quarter-wave polarizer to change the polarization of incident wave[8], etc.

In th is work, our main focus is to design Frequency Selective Surface (FSS) absorber to provide security for WLAN signals. As WLANs are based on radio frequency (RF), the information which transmitted from one point to another can be hacked or tapped by intruders. A band-stop filter can be used to provide security, but it can attenuate other useful microwave signals. So, there is a need of such

* Corresponding author:

umair-rafique@hotmail.com (Umair Rafique)

Published online at http://journal.sapub.org/ijea

Copyright (C) 2012 Scientific \& Academic Publishing. All Rights Reserved type of filter which only provides attenuation for the desired frequencies and allow other communication bands to pass through it. This can be done by the designing of FSS absorber. The design and configuration of FSS absorber was first presented by Salisbury and Jaumann[9-12]. The presented designs are much easier to model, but the problem associated with the designs is the unwanted attenuation to other communication bands. To overcome this problem, many researchers proposed different techniques and designe d FSS absorbers for different mic rowave applications.

In[13], authors presented FSS absorber for mm-wave applications. They used full ground plane instead of conducting FSS to get absorption for wide range of frequencies. But, the presented results showed that it can attenuate communication bands other than the band of interest, because the ground plane is directly printed on the bottom of dielectric. Also, the stability characteristics of the presented design were not checked at oblique incidences which are the major problem in the designing of FSS absorber. In[14], authors presented same kind of FSS absorber as that of[13] for single band operation. They used lossy dielectric slab and due to the use of this technique they got good frequency stability at different incident angles. Also, the transmission of other communication bands is not disturbed.

As discussed above, stability is one of the major problems in designing of FSS absorber. In the last decade, researchers proposed different techniques to get rid of this problem. In[15], authors presented a simple fractal FSS structure to get 
frequency stability. But, they have designed different structures for different incident angles. In[16-17], authors presented fractal shaped band-stop and band-pass FSS to get stability at maximum angle of incidence. They have presented good frequency stability, but the presented designs are difficult to model and fabricate. In[18], a simple FSS structure based on cross-dipole elements was presented. Authors used Genetic Algorithm (GA) optimization to get a novel design. Analytically this is a good approach, but its practical implementation not yet available. Also, the above discussed designs are only associated with band-stop and band-pass characteristics.

The FSS absorber for WLAN security was first time presented in[19]. The overall design configuration is based on the principle of Salisbury and Jaumann absorber[9-12]. After that, in[20], the oblique incidence performance of FSS absorber design was presented which was the modification in[19]. Maximum frequency stability and absorption rate was demonstrated by sandwiching the conducting FSS element between two dielectrics. But the presented design lost its functionality at TM $45^{\circ}$ angle of incidence because the conducting FSS element was not able to function properly due to some air gap between the layers. Also, it is hard to fabricate such kind of configuration. In[21], authors presented simple and compact FSS absorber design for WLAN security. But, the problem remains the same that for TM $45^{0}$ the absorption is not band limited.

In this paper, a novel FSS absorber design is presented for $2.45 \mathrm{GHz}$ and $5.25 \mathrm{GHz}$ WLAN security. The proposed design has the ability to absorb WLAN signals at better rate. Also, the stable frequency response is achieved up to $45^{\circ}$ oblique angle of incidence for both TE and TM polarizations. The presented design is fully obeying the principle of Salisbury and Jaumann absorber design and also the distance between the conducting and resistive sheets is managed according to the criteria[9-12].

The remainder of the paper is as follows. Section 2 describes the design and configuration of dual-band FSS absorber. Section 3 describes the results and discussion on the results. Finally, we conclude the paper in Section 4.

\section{Design and Configuration of Dual-Band FSS Absorber}

This section describes the design and configuration of dual-band FSS absorber. The overall design parameters and configuration is shown in Fig. 1. First, a conducting sheet which consists of two square-loop elements is designed to demonstrate the reflection properties of dual-band FSS design. The distance between the two conducting elements is equal to $\lambda_{\mathrm{o}} / 2$. The outer square-loop element is tuned to 2.45 $\mathrm{GHz}$ and inner square-loop element is tuned to $5.25 \mathrm{GHz}$, respectively. The dimensions of both the elements are shown in Fig. 1. The dielectric used for the design purpose is FR-4 having dielectric constant $\varepsilon_{\mathrm{r}}=4.4$ and thickness $1.6 \mathrm{~mm}$. The function of conducting FSS layer is to provide reflection for WLAN signals while passing other useful micro wave signals. The square-loop elements are designed according to the formu la which is given below

$$
L=W=\frac{c}{2 f_{r} \sqrt{\varepsilon_{\text {eff }}}}
$$

where,

$$
\varepsilon_{\text {eff }}=\sqrt{\frac{\varepsilon_{r}+1}{2}}
$$

where, $L$ is the length of square-loop element, $W$ is the width of square-loop element, $c$ is the speed of light in free-space, $f_{r}$ is the resonant frequency, $\varepsilon_{r}$ is the dielectric constant of the material and $\varepsilon_{e f f}$ is the effective dielectric constant of the material, respectively.

After that, the absorption characteristics are achieved by placing resistive sheet in-front of the conducting sheet at a distance of $12 \mathrm{~mm}$. This design configuration follows the principle of Salisbury and Jaumann absorber[9-12]. FR-4 is also used for the designing of resistive FSS elements having thickness $0.8 \mathrm{~mm}$. The resistivity of the resistive layer is chosen to be $50 \Omega /$ square. Other resistance values are also available, but its use can attenuate other communication bands which is not useful in our case[19]. The dimensions of resistive layer and FSS elements are shown in Fig. 1.

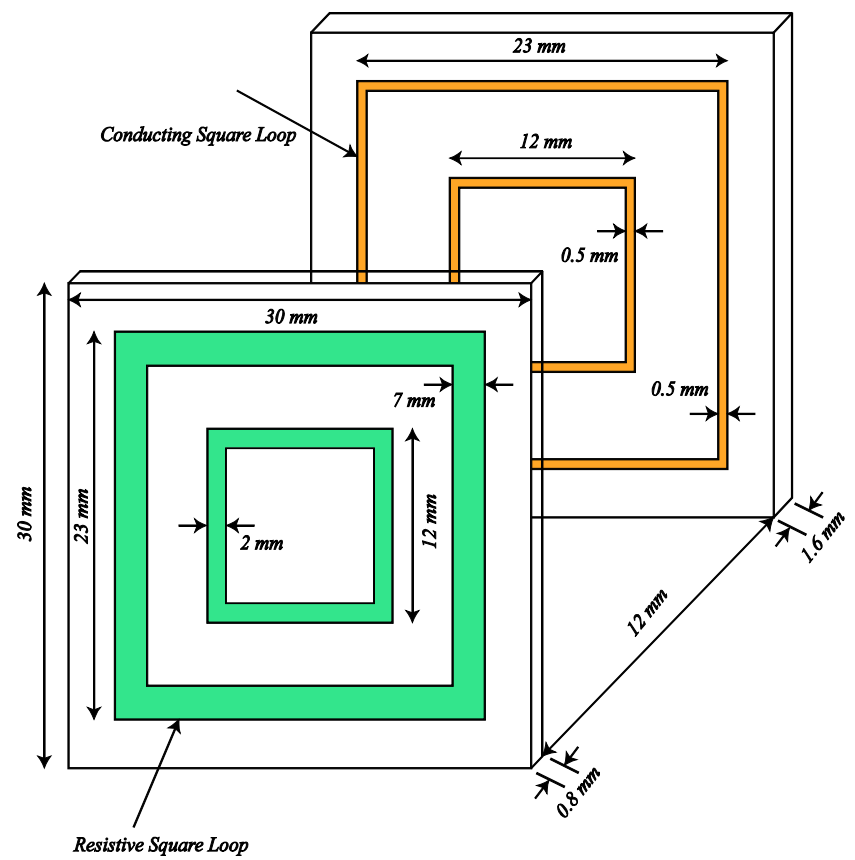

Figure 1. Design and configuration of dual-band Frequency Selective Surface (FSS) absorber

\section{Simulation Results and Discussions}

To demonstrate the above described design, a simulation setup is set by using Ansoft HFSS. The simulations are done

by applying real time boundary conditions. The results for perpendicular (TE) and parallel (TM) polarizations for $0^{0}$ and $45^{\circ}$ oblique incidences are presented here.

First of all, conducting FSS layer which consists of two 
square-loop elements are simulated to demonstrate the reflection properties of FSS design. Fig. 2 shows the reflection and transmission coefficients of a stable conducting FSS layer at TE polarization. For $0^{0}$ and $45^{\circ}$ oblique angle of incidence, the resonant frequencies are 2.42 $\mathrm{GHz}, 5.22 \mathrm{GHz}, 2.41 \mathrm{GHz}$ and $5.25 \mathrm{GHz}$, respectively. The transmission coefficients corresponding to the resonant frequencies are $-34.1 \mathrm{~dB},-29.5 \mathrm{~dB},-35.7 \mathrm{~dB}$ and $-33.08 \mathrm{~dB}$, respectively. The reflection coefficients at the resonant frequencies are almost $0 \mathrm{~dB}$ which means that maximum reflection occurred in the bands of interest. Fig. 3 shows the reflection and transmission coefficient results for TM polarization. For $0^{0}$ and $45^{\circ}$ oblique angle of incidence, the resonant frequencies are $2.48 \mathrm{GHz}, 5.24 \mathrm{GHz}, 2.51 \mathrm{GHz}$ and $5 \mathrm{GHz}$, respectively. The transmission coefficients corresponding to the resonant frequencies are $-33.7 \mathrm{~dB},-29.8$ $\mathrm{dB},-30.8 \mathrm{~dB}$ and $-25.5 \mathrm{~dB}$, respectively. In this case, the reflection coefficient at the resonant frequencies is almost 0 dB.

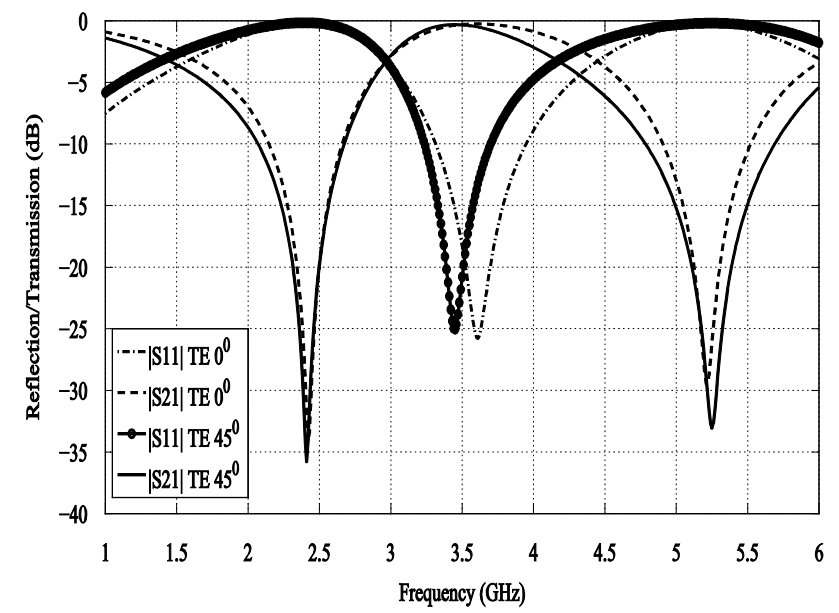

Figure 2. Reflection and transmission coefficients of conducting FSS layer for TE polarization

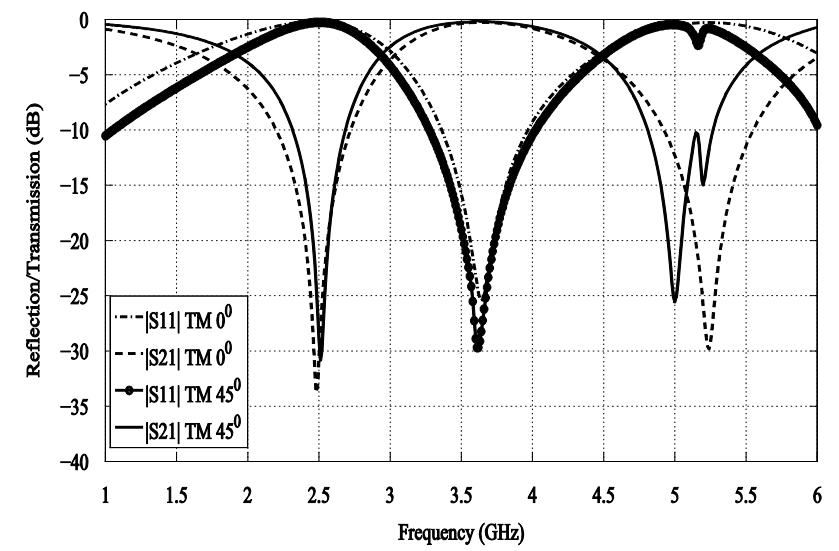

Figure 3. Reflection and transmission coefficients of conducting FSS layer for TM polarization

After that, resistive FSS layer is placed in-front of the conducting FSS layer at a distance of $12 \mathrm{~mm}$ to demonstrate the absorption characteristics of FSS design. Fig. 4 shows the simulated results of dual-layer FSS absorber for TE polarization. The resonant frequencies for $0^{0}$ and $45^{0}$ oblique angle of incidence are $2.5 \mathrm{GHz}, 5.23 \mathrm{GHz}, 2.52 \mathrm{GHz}$ and $5.10 \mathrm{GHz}$, respectively. In this case, only absorption results are presented. The absorption coefficients noted at the resonant frequencies are $-44.7 \mathrm{~dB},-8.5 \mathrm{~dB},-14.3 \mathrm{~dB}$ and -7.4 $\mathrm{dB}$, respectively. After that, the dual-layer FSS absorber is simulated for TM polarization because one polarization mode is not enough to demonstrate the stability characteristics of FSS absorber. Fig. 5 shows the absorption characteristics of dual-layer FSS absorber for TM polarization. For $0^{0}$ and $45^{\circ}$ oblique incidences, the resonant frequencies are $2.52 \mathrm{GHz}, 5.15 \mathrm{GHz}, 2.51 \mathrm{GHz}$ and 5.15 $\mathrm{GHz}$, respectively. The absorption coefficients at the resonant frequencies are $-48.5 \mathrm{~dB},-8 \mathrm{~dB},-47 \mathrm{~dB}$ and $-8 \mathrm{~dB}$, respectively.

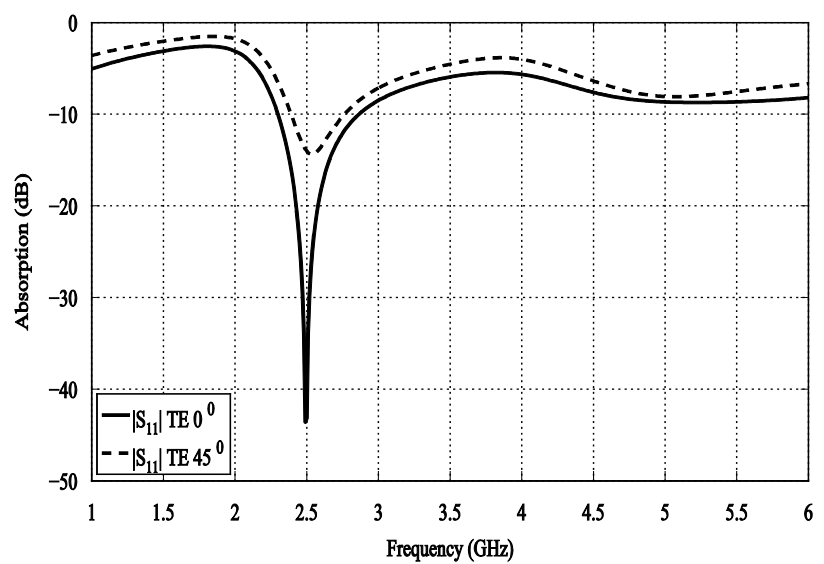

Figure 4. Absorption characteristics of dual-layer FSS absorber for TE polarization

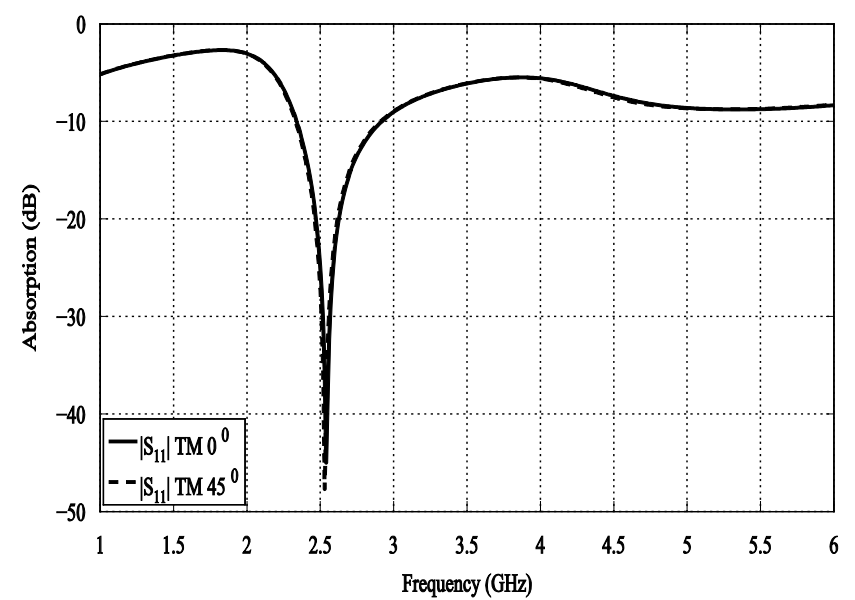

Figure 6. Absorption characteristics of dual-layer FSS absorber for TM polarization

Above presented results demonstrated that the proposed dual-band FSS absorber design has the ability to absorb WLAN signals. Also, the proposed FSS design has a stable frequency response for different oblique incidences for TE and TM polarizations which is better than the previous reported data[20-21]. The proposed design is easy to model and fabricate. One thing noted from the results, that the absorption level is greater at $2.45 \mathrm{GHz}$ than $5.25 \mathrm{GHz}$. Because, there is a mutual coupling between FSS elements and also the distance between the FSS elements is less and 
their wavelengths are interacting with each other. For future work, the mutual coupling can be reduced by applying an aperture type FSS between the elements.

\section{Conclusions}

A dual-band Frequency Selective Surface (FSS) absorber is presented for WLAN security. The FSS absorber design is based on two FSS layers which consist of two square-loop elements tuned to $2.45 \mathrm{GHz}$ and $5.25 \mathrm{GHz}$, respectively. The proposed FSS design provides maximum stability for the desired WLAN frequency bands for both perpendicular (TE) and parallel (TM) polarizations when the incident wave angle changed from $0^{0}$ to $45^{0}$. The performance of design is demonstrated through real time simulation and it is noted, that the FSS design will be able to absorb desired frequency bands for the wide range of incidence angles. Research is in progress to achieve maximum absorption rate for WLA N upper band ( $5.25 \mathrm{GHz})$ with the reduction in distance between two layers.

\section{REFERENCES}

[1] Munk, B. A., Frequency Selective Surface: Theory and Design, New York: Wiley, 2000.

[2] Virone, G., Tascone, R., Addamo, G. and Peverini, O. A., “A design strategy for large dielectric radome compensated joints”, IEEE Antennas and Wireless Propagation Letters, vol. 8, pp. 546-549, 2009.

[3] Zadeh, A. K. and Karlsson, A., "Capacitive circuit method for fast and efficient design of wideband radar absorbers”, IEEE Transactions on Antennas and Propagation, vol. 57, no. 8, pp. 2307-2314, 2009.

[4] Munk, B. A., Munk, P. and Pryor, J., “On designing circuit and analog absorbers (CA absorbers) for oblique angle of incidence”, IEEE Transactions on Antennas and Propagation, vol. 55, no. 1, pp. 186-193, 2007.

[5] Bao, X. L., Ruvio, G., Ammann, M. J. and Jhon, M., “A novel GPS patch antenna on a fractal Hi-impedance surface substrate”, IEEE Antennas and Wireless Propagation Letters, vol. 5, pp. 323-326, 2006.

[6] Rafique, U., Ahmed, M. M., Haq, M. A. and Rana, M. T., "Transmission of RF signals through energy efficient window using FSS”, Proceedings of the $7^{\text {th }}$ International Conference on Emerging Technologies, pp. 1-4, 2011.

[7] Suwailam, M. M. B., Siddique, O. F. and Ramahi, O. M., "Mutual coupling reduction between microstrip patch antennas using slotted-complementary split-ring resonator”, IEEE Antennas and Wireless Propagation Letters, vol. 9, pp. 876-878, 2010.
[8] Kiani, G. I. and Dyadyuk, V., "Quarter-wave polarizer based on frequency selective surface", $40^{\text {th }}$ European Microwave Conference, pp. 1361-1364, 2010.

[9] Knott, E. F. and Lunden, C. D., "The two-sheet capacitive Jaumann absorber", IEEE Transactions on Antennas and Propagation, vol. 43, no. 11, pp. 1339-1343, 1995.

[10] Fante, R. L. andMcCormack, M. T., “Reflection properties of the Salisbury screen”, IEEE Transactions on Antennas and Propagation, vol. 36, no. 10, pp. 1443-1454, 1998.

[11] Abdelaziz, A. A., "A novel technique for improving the performance of Salisbury screen”, $17^{\text {th }}$ National Radio Science Conference, 2000.

[12] Haupt, R. L., “Scattering from small Salisbury screens”, IEEE Transactions on Antennas and Propagation, vol. 54, no. 6, pp. 1807-1810, 2006.

[13] Sakran, F. Oz, Y. N., Ron, A., Golosovsky, M., Davidov, D. and Frenkel, A., "Absorbing frequency-selective-surface for the mm-wave range”, IEEE Transactions on Antennas and Propagation, vol. 56, no. 8, pp. 2649-2655, 2008.

[14] Zhao, H., Wan, G. and Wan, W., “Absorbing properties of frequency selective surface absorbers on a lossy dielectric slab”, Progress In Electromagnetic Research Symposium, pp. 165-168, 2009.

[15] Romeu, J. and Samii, Y. R., "Fractal FSS: A novel dual-band frequency selective surface”, IEEE Transactions on Antennas and Propagation, vol. 48, no. 7, pp. 1097-1105, 2000.

[16] Zhang, J. C., Yin, Y. Z. and Ma, J. P., "Frequency selective surfaces with fractal four legged elements”, Progress In Electromagnetic Research Letters, vol. 8, pp. 1-8, 2009.

[17] Xue, J.-Y., Gong, S.-X., Zhang, P.-F., Wang, W. and Zhang, F.-F., "A new miniaturized fractal frequency selective surface with excellent angular stability”, Progress In Electromagnetic Research Letters, vol. 13, pp. 131-138, 2010.

[18] Parker, E. A., Chuprin, A. D., Batchelor, J. C. and Savia, S. B., "GA optimization of crossed dipole FSS array", Electronics Letters, vol. 37, no. 16, pp. 996-997, 2001.

[19] Kiani, G. I., Wiley, A. R. and Esselle, K. P., "Frequency selective surface absorber using resistive cross-dipoles”, IEEE Antennas and Propagation Society International Symposium, pp. 4199-4202, 2006.

[20] Kiani, G. I., Ford, K. L., Esselle, K. P., Wiley, A. R. and Panagamuwa, C. J., "Oblique incidence performance of a novel frequency selective surface absorber", IEEE Transactions on Antennas and Propagation, vol. 55, no. 10, pp. 2931-2934, 2007.

[21] Rafique, U., Kiani, G. I., Ahmed, M. M. and Habib, S., "Frequency selective surface absorber for WLAN security", Proceedings of the $5^{\text {th }}$ European Conference on Antennas and Propagation, pp. 872-875, 2011. 